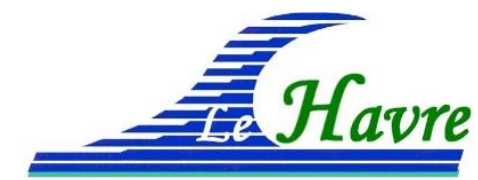

XVI ${ }^{\text {èmes }}$ Journées Nationales Génie Côtier - Génie Civil

Le Havre, 2020

DOI:10.5150/jngcgc.2020.004 C Editions Paralia CFL

disponible en ligne - http://www.paralia.fr - available online

\title{
La notion de quantité de mouvement : Son influence dans la prise en compte des actions de la houle
}

Partie A - Effet de claque d'une vague sur une paroi verticale

Partie B - Écoulements générés par la houle au sein de fonds sableux

\section{Michel BÉLORGEY ${ }^{1}$}

\author{
1. Association POSEIDOM, Parc du Chapitre, 76420 Bihorel, France. \\ belorgey@poseidom.fr
}

\section{Résumé :}

Avec le développement des moyens d'instrumentation actuels permettant la mesure instantanée des vitesses au sein d'un écoulement généré par la houle (LDV et VDU), il est possible, depuis 1982/1983, d'aborder plus efficacement les phénomènes physiques liés à l'action de la houle, tant en ce qui concerne l'action des vagues déferlantes sur les ouvrages (Partie A), que l'influence de la houle sur les écoulements qu'elle génère sur les fonds marins perméables et l'érosion qui en découle (Partie B). En ce qui concerne la Partie A et l'effet de claque d'une vague sur une paroi verticale, les mesures de vitesses que nous avons effectuées au Laboratoire par Vélocimétrie Laser, montrent que si l'impact se produit au moment précis où la vague commence à déferler, celle-ci est brusquement stoppée dans sa propagation. L'effort qui en résulte sur la paroi est donc dû à la variation de quantité de mouvement (c'est un choc). La masse de la vague (tout ou partie) étant grande, l'effort est donc très important. Nous en présentons les résultats et ils permettent de comprendre la formation des amas de blocs cyclopéens sur l'île de Banneg (archipel de Molène en Bretagne, GUILCHER, 1959), voir figure A en annexe. En ce qui concerne la partie $B$ et les écoulements générés par la houle ou les courants marins au sein d'un fond perméable les mesures des vitesses interstitielles que nous avons effectuées par VDU, en canal au sein d'un fond sableux, tant dans la zone de swash des vagues sur les plages, que dans le cas d'un écoulement permanent, donnent des résultats analogues à ceux qui avaient été obtenus dans le cas d'un transfert de chaleur d'une source thermique, au sein d'une couche limite, sur une paroi absorbante (1978). Ceci nous a conduits à reconsidérer l'équation de transfert d'une grandeur scalaire au sein d'un écoulement. Équation de Navier-Stockes pour la quantité de mouvement $(\rho U)$ et Corrsin pour l'enthalpie $\left(\rho C_{p} \theta\right)$. Et nous a permis de définir pour la quantité de mouvement, au sein d'un lit poreux, une grandeur $\left(\left(b_{f l}\right)\right.$ différente de la notion de perméabilité et analogue à la diffusivité en thermique $\left(b_{t h}\right)$.

\section{Mots-clés :}

Génie Côtier, Action de la houle sur les ouvrages, Érosion sédimentaire, Vitesses interstitielles. 


\section{Thème 1 - Hydrodynamique côtière}

\section{Partie A - Effet de claque d'une vague sur une paroi verticale}

\section{A.1. Introduction}

Les premières études sur le sujet ont été entreprises à l'Institut Franzius de Hanovre par les Professeurs H. W. Partensky et H. Oumeraci (Professeur à l'Université de Braunschweig). Elles ont été poursuivies dans le cadre du programme Européen PROVERBS de MAST III. (PRObabilistic design tools for VERtical BreakwaterS. MAS3-CT95-0041, 1996-1999) auquel nous avons participé.

Et en ce qui concerne le sujet de l'effet de claque d'une vague déferlante sur une paroi verticale, bien que conscient du phénomène, il était difficile de partager l'idée que c'était la bulle d'air emmagasinée lors du déferlement (plongeant) qui était l'élément principal. Sans a priori, remettre en cause son existence.

Mais, avec l'acquisition d'un deuxième canal à houle (1998), à parois vitrées comme le premier, mais muni d'un générateur de houle spécifique permettant de programmer des vagues déferlantes à une distance bien précise dans le canal (et donc sur une paroi verticale placée dans le canal), il a été possible de valider la théorie selon laquelle c'est bien la variation de quantité de mouvement de la vague, au moment de l'impact, qui est à prendre en compte.

L'effet de claque d'une vague déferlante sur une paroi est dû au fait que la masse de la vague déferlante est brutalement stoppée. Imaginez un train percutant un mur !

\section{A.2. Dispositif expérimental}

\section{A.2.1 Le canal à houle}

Les études ont été réalisées dans le canal que nous avons conçu et mis au point en 1998 en collaboration avec le bureau d'études écossais Edinburgh Designs Ltd. Ce canal de $22 \mathrm{~m}$ de long et $0.80 \mathrm{~m}$ de large, a des parois vitrées pour les mesures par vélocimétrie laser. Le batteur est un batteur piston, commandé par micro-ordinateur et servomoteur. Il est lui aussi le fruit de la coopération avec ce même bureau d'études, il permet la génération d'ondes complexes : houles aléatoires à partir de divers spectres classiques (Pierson-Moskowitz, Jonswap, Bretschneider, etc...) ou personnalisées (en fonction d'études in situ), déferlement et/ou impact par train d'ondes ...

Les mesures des vitesses ont été réalisées par vélocimétrie laser (DANTEC), système de mesure non intrusif. Cette technique adaptée à la houle en 1982 (première mondiale) a été très bien détaillée dans les diverses publications associées aux travaux du Professeur Bélorgey (BÉLORGEY \& LE BAS, 1984 ; BÉLORGEY et al., 1989 \& 2000 ; MIHOUBI et al., 2008). 


\section{XVI'̀mes Journées Nationales Génie Côtier - Génie Civil \\ Le Havre 2020}

\section{A.2.2 La maquette}

A la fin des années 90, nous effectuions des études concernant la mesure des pressions générées par la houle sur les parois verticales d'un caisson JARLAN dans le cadre du programme Européen PROVERBS de MAST III auquel nous participions.

Nous avons donc utilisé la maquette réalisée pour ces études en modélisation physique à l'échelle $1 / 25^{\text {ème }}$. Par ailleurs, les parois verticales du caisson JARLAN étaient munies de capteurs de pression.

\section{A.3. Analyse des résultats}

Nous avons enregistré les mesures de pression du capteur $\mathrm{M}_{3}$ situé sur la face avant de la paroi perforée. Les mesures des vitesses ont été enregistrées aux points $M_{1}$ et $M_{2}$, voir figure 1. La figure 2, représente les vitesses lors l'impact d'une vague déferlante. Les figures 3(a) et 3(b) représentent l'enregistrement simultané des vitesses au point $\mathrm{M}_{1}$ et $\mathrm{M}_{2}$ associées aux pressions enregistrées par le capteur $\mathrm{M}_{3}$.
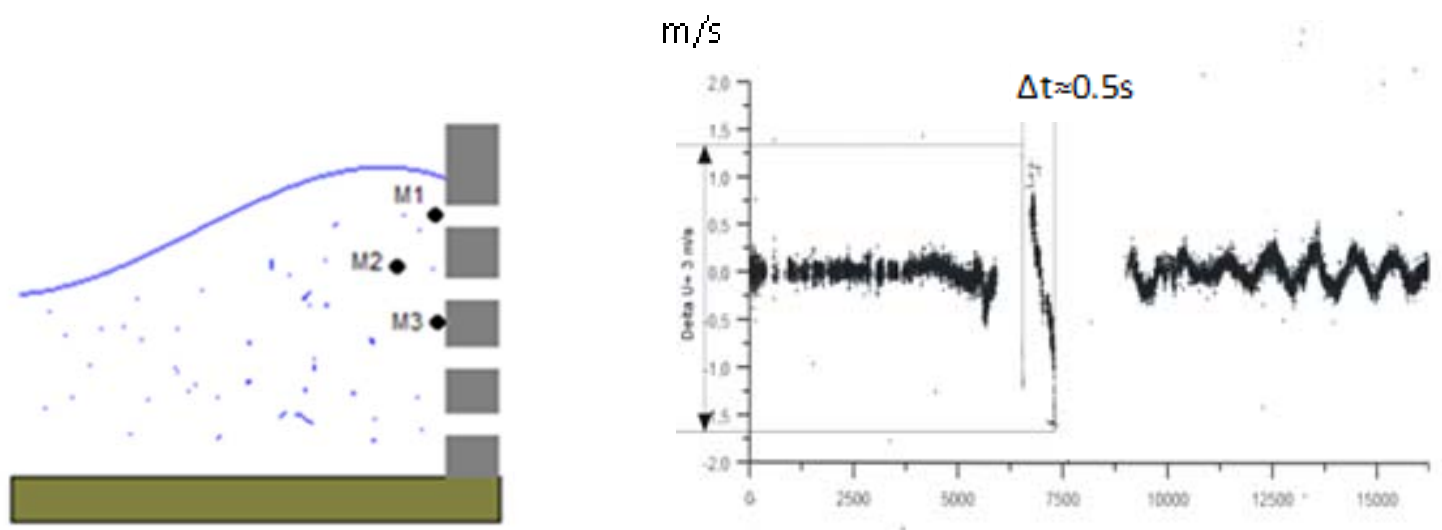

Figure 1. Position des points de mesure. Figure 2. Évolution des vitesses lors de l'impact.

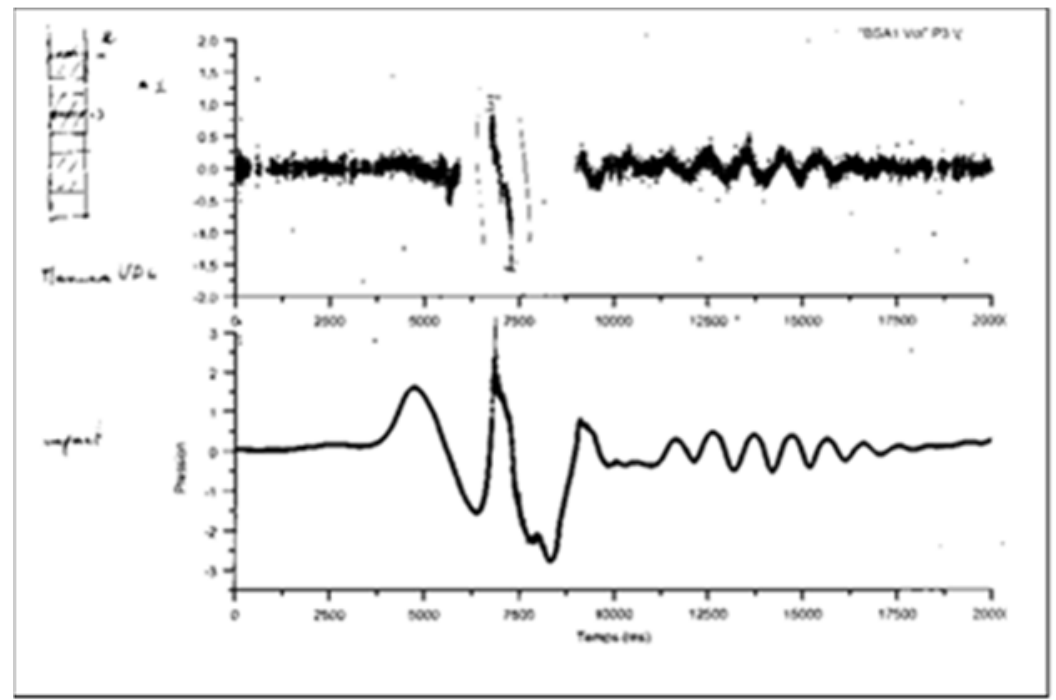

Figure 3. Enregistrement simultané des vitesses et des pressions lors de l'impact. 


\section{Thème 1 - Hydrodynamique côtière}

Les mesures ont été réalisées en 2002 dans le cadre du stage de DEA (Génie Côtier) de N. Hadj-Rabia. Le responsable de son stage était J.-M. Rousset. De ces mesures et des visualisations associées (voir photos) il nous est possible de proposer la théorie suivante : Au moment de l'impact d'une vague "déferlante" (figure 4) :

- La vitesse des particules d'eau de la partie supérieure de la vague a une composante verticale "négative".

- La vitesse des particules d'eau de la partie inférieure de la vague a une composante "positive".

- L'eau étant incompressible, ces deux composantes s'opposent et bloquent instantanément (mais pas de façon persistante) toute évolution de la vague sur une durée très courte.

- Cet effet de blocage "instantané" de la vague déferlante, sans run-up est visible sur la photo de la figure 4.

- Ce n'est qu'après cette phase très courte, et compte tenu des vitesses des particules d'eau qui suivent, que la vague reprend son mouvement, elle peut générer une gerbe d'eau au droit de la paroi, qui à mon avis n'est pas exactement un run-up.

Par contre, dans le cas où l'impact ne correspond pas à l'instant précis du déferlement sur la paroi, il se produit un phénomène de run-up très visible sur la photo de la figure 5.
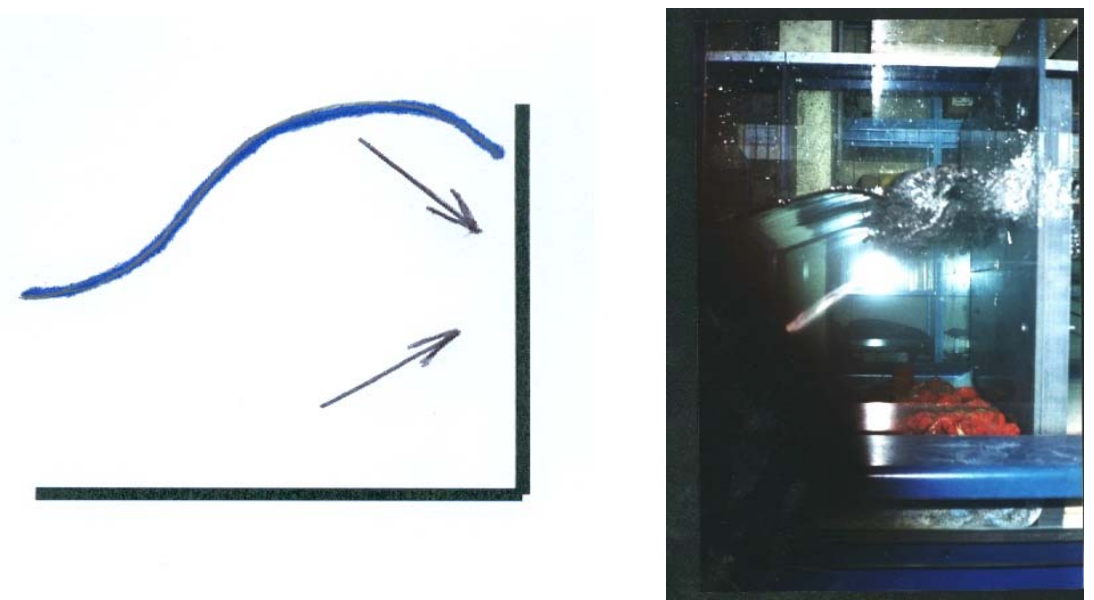

Figure 4. Schéma et photo illustrant la dynamique de l'impact d'une vague déferlante. 


\section{XVI'̀mes Journées Nationales Génie Côtier - Génie Civil \\ Le Havre 2020}
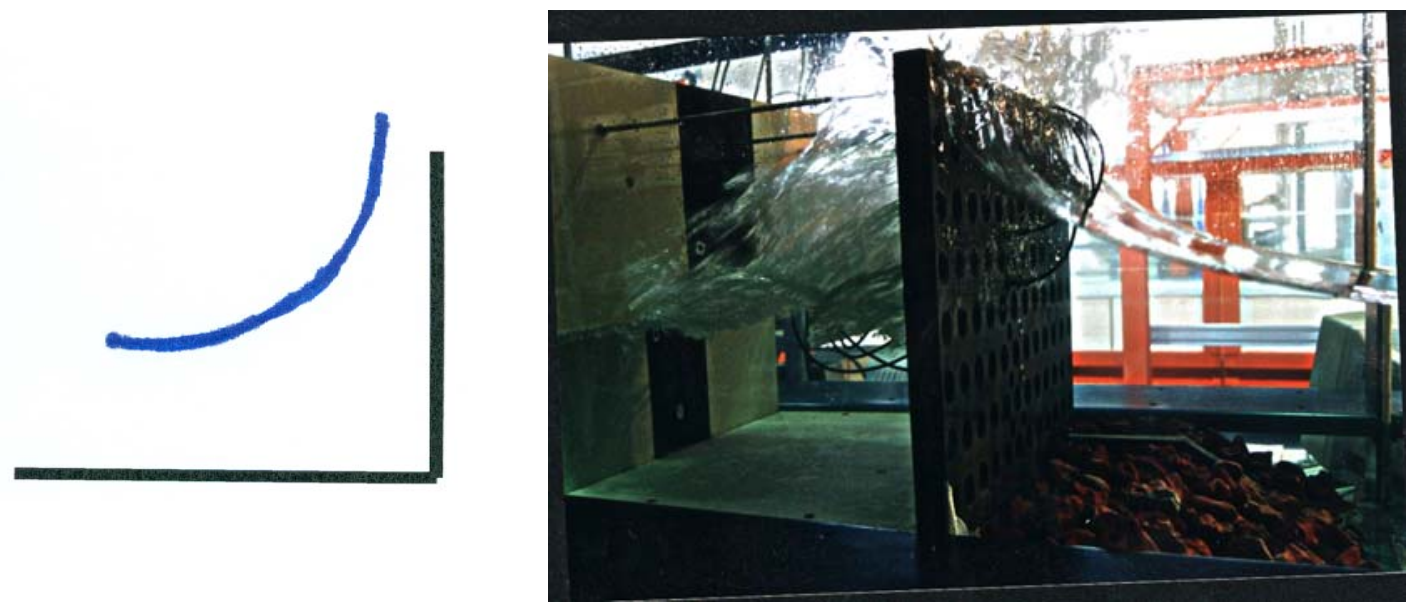

Figure 5. Schéma et photo illustrant la dynamique du run-up.

\section{A.4. Quantification des efforts}

L'enregistrement des variations de vitesse au moment de l'impact (figure 2) nous donne les résultats suivants :

- Variation de vitesse au moment de l'impact : $\Delta U \approx 3 \mathrm{~m} / \mathrm{s}$

- Durée du choc : $\Delta t \approx 0,5 \mathrm{~s}$

- D'où une décélération correspondante : $\Gamma=\Delta U / \Delta t \approx 6 \mathrm{~m} / \mathrm{s}^{2}$.

La percussion due à l'effet de claque d'une vague sur la paroi produit une force égale à : $F=m . \Gamma$, (m étant la masse de la vague à prendre en compte)

Dans le cas particulier de notre étude (largeur de canal $l=0,8 \mathrm{~m}$; longueur d'onde $L=1,6 \mathrm{~m}$ (prendre $L / 2$ ); profondeur d'eau $p=0,6 \mathrm{~m}$ ), même en introduisant un coefficient pondérateur pour ne prendre en compte qu'une partie de la masse de la vague, l'effort est considérable : $F=(k) .2304 \mathrm{~N}$, avec $k$, coefficient pondérateur de masse.

\section{A.5. Conclusion}

L'effet de claque de la vague a été étudié sur la paroi extérieure d'un caisson JARLAN, c'est-à-dire sur une paroi perforée. Or, si l'effort généré par l'impact était dû à l'existence d'une bulle d'air, celle-ci se serait échappée par les orifices de la paroi.

Donc l'effet de claque d'une vague est dû à une variation de quantité de mouvement et non à la compressibilité d'une bulle d'air emmagasinée par la vague au moment du déferlement.

Par contre, il est nécessaire d'avoir une estimation la plus précise possible de la valeur du coefficient $\mathrm{k}$ pour connaître la quantité de la masse de la vague à prendre en compte :

- Analyse de la variation instantanée du champ des vitesses sur toute la masse de la vague au moment de l'impact (elle n'est peut être pas identique en chaque point de la vague)

- Étude de l'impact pour différentes conditions de houle déferlante. 


\section{Thème 1 - Hydrodynamique côtière}

\section{Partie B - Écoulements générés par la houle au sein de fonds sableux}

\section{B.1. Introduction}

Les processus physiques associés au transport sédimentaire n'ont été abordés jusqu'à maintenant qu'à partir d'analyses du champ des vitesses au sein de la veine fluide. La plupart des modèles ont été établis pour des écoulements sur fond imperméable.

Le but de notre étude est d'exploiter la technique ultrasonore (VDU - Vélocimétrie Doppler Ultrasonore) pour analyser le champ des vitesses au sein d'un écoulement permanent sur un fond sédimentaire poreux. Les vitesses sont mesurées pour plusieurs lits sédimentaires, aussi bien au sein de la veine fluide qu'au sein du sédiment. Nos résultats mettent en évidence :

- Une répartition exponentielle des vitesses à l'intérieur du sédiment.

- Une discontinuité de vitesse à l'interface eau/sédiment, entre la vitesse dans l'écoulement libre et la vitesse au sein du sédiment.

Ces résultats mettent en évidence la nécessité de baser l'analyse du transport sédimentaire sur la réalité des processus physiques à l'interface eau/sédiment.

Afin de mieux comprendre les processus physiques associés à ce phénomène, nous avons adapté la technique de Vélocimétrie Doppler Ultrasonore (VDU) à la mesure instantanée des vitesses interstitielles locales au sein d'une masse poreuse.

\section{B.2. Dispositif expérimental et calibration de la technique des mesures}

Le dispositif est constitué d'un canal d'une longueur totale de 3,5 m et de section rectangulaire de $(0,10 \mathrm{~m} \times 0,25 \mathrm{~m})$, permettant d'effectuer des mesures de calibration de vitesse par le vélocimètre Doppler ultrasonore (figure 6). Les parois et le fond du canal sont vitrés d'une épaisseur de $10 \mathrm{~mm}$, permettant ainsi de faire des observations et mesures optiques,

Nous avons matérialisé le lit sédimentaire par différents matériaux, mousses alvéolaires, billes de verre, sables, présentant des structures et porosités différentes. L'épaisseur du lit variant entre $50 \mathrm{~mm}$ et $75 \mathrm{~mm}$.

Le canal est alimenté par un réservoir à charge constante en matériau PVC de section carrée de coté $0,30 \mathrm{~m}$ et $0,25 \mathrm{~m}$ de hauteur. L'arrivée d'eau se fait par un système de crépine, l'eau est tranquillisée par des plaques en géo membrane d'environ $5 \mathrm{~cm}$ d'épaisseur. Dans le canal, le débit est contrôlé par un seuil réglable, le trop-plein d'eau est évacué vers le réservoir d'alimentation du canal. L'alimentation du canal est assurée par un circuit fermé au moyen d'une pompe de débit maximal 5,0 1/s. Pour une meilleure linéarisation de l'écoulement et afin de réduire les effets de turbulence, deux cages de nid d'abeilles ont été installées avant la sortie du convergent. Les débits d'écoulement sont mesurés par un débitmètre triangulaire à seuil mince situé à la sortie du canal, après un étalonnage par la méthode de mesure volumétrique. 


\section{XVİ̀mes Journées Nationales Génie Côtier - Génie Civil \\ Le Havre 2020}

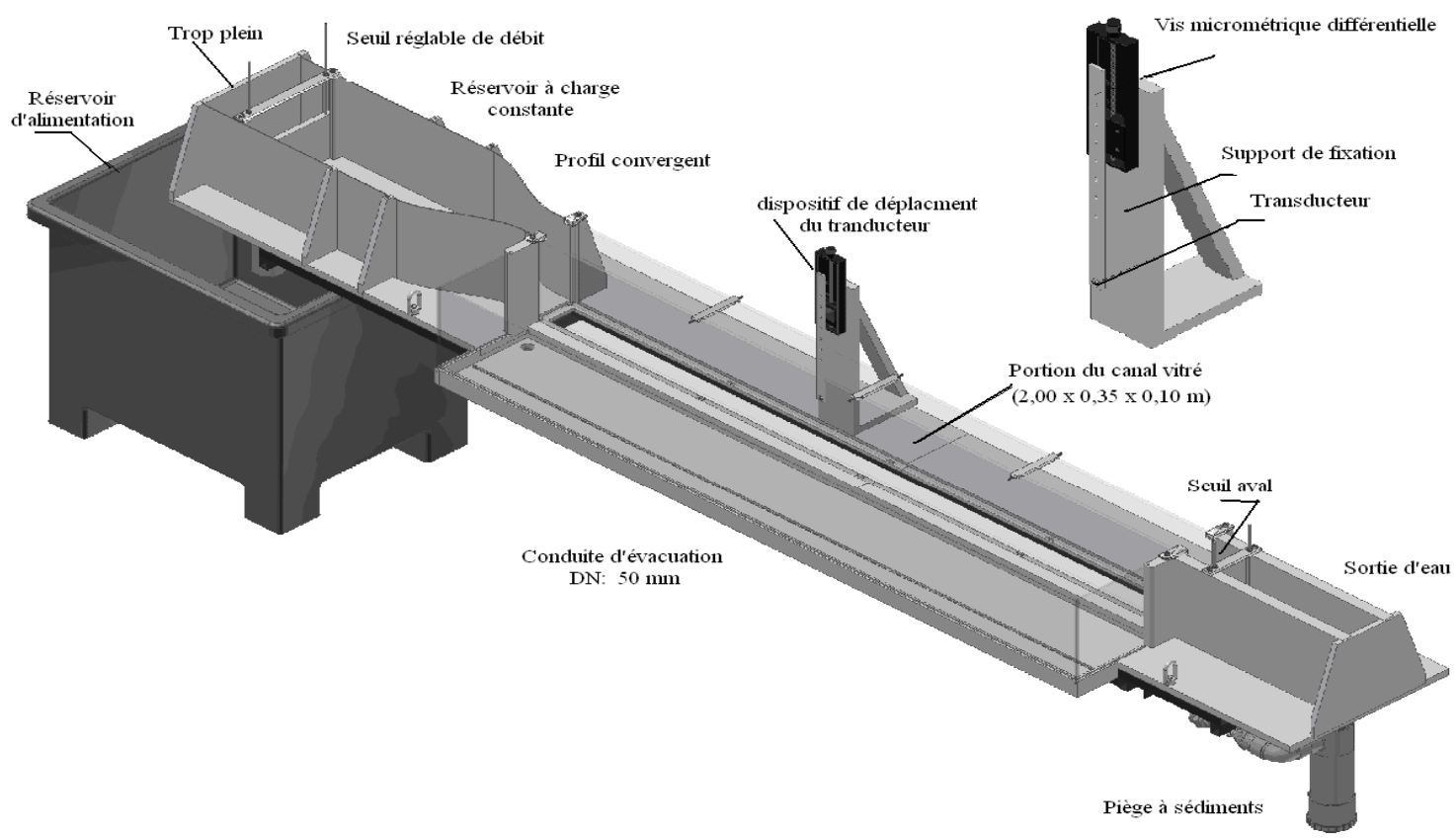

Figure 6. Dispositif expérimental.

\section{B.3. Analyse des résultats expérimentaux en écoulement permanent}

Sur les figures $7 \mathrm{a}$ et $7 \mathrm{~b}$, nous avons représenté les évolutions des vitesses pour différents lits sédimentaires et différents débits en fonction de l'axe $z$ (profondeur pour les sédiments et hauteur d'eau pour la veine fluide). La cote " 0 " correspond à l'interface eau/sédiment.

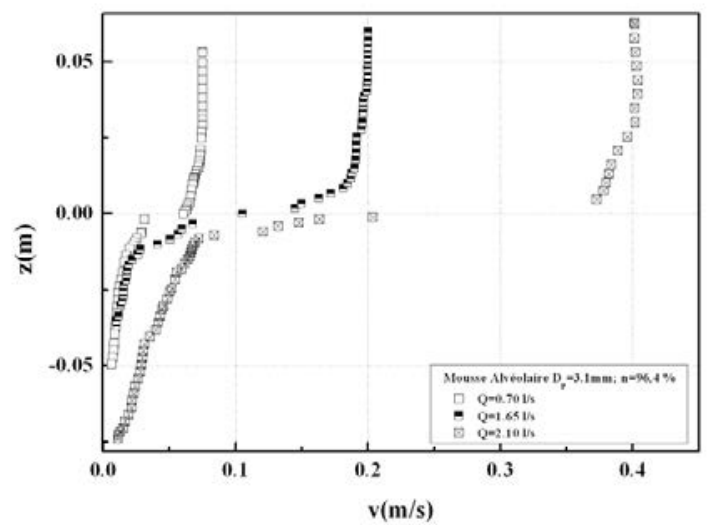

Figure 7a. Résultats correspondant à un lit sédimentaire constitué de mousse alvéolaire $\left(D_{p}=3.1 \mathrm{~mm} ; n=96.4 \%\right)$.

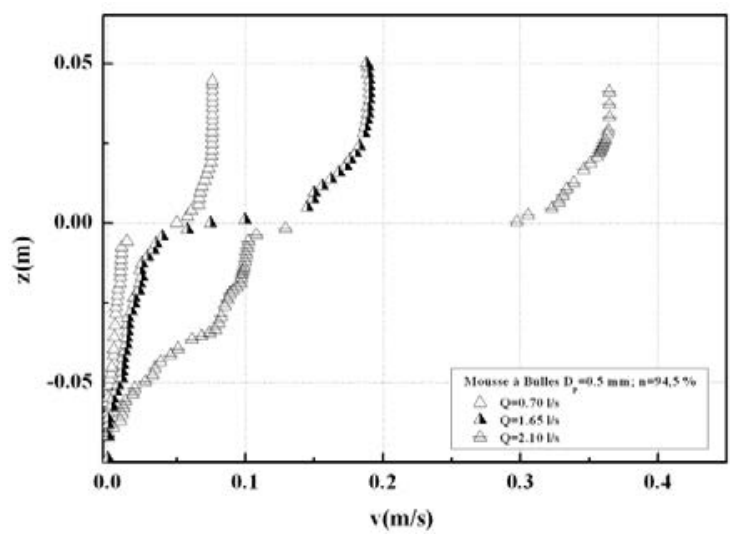

Figure $7 b$. Résultats correspondant à un lit sédimentaire constitué de mousse à bulles $\left(D_{p}=0.5 \mathrm{~mm} ; n=94.5 \%\right)$. 


\section{Thème 1 - Hydrodynamique côtière}

Comme le montre en détail la figure 8 (mousse alvéolaire), ces résultats mettent en évidence :

- Une "discontinuité" des vitesses à l'interface eau/sédiment, c'est-à-dire un gradient très important fonction du débit et de la nature du sédiment.

- Une vitesse non nulle sur le fond (pour la veine fluide).

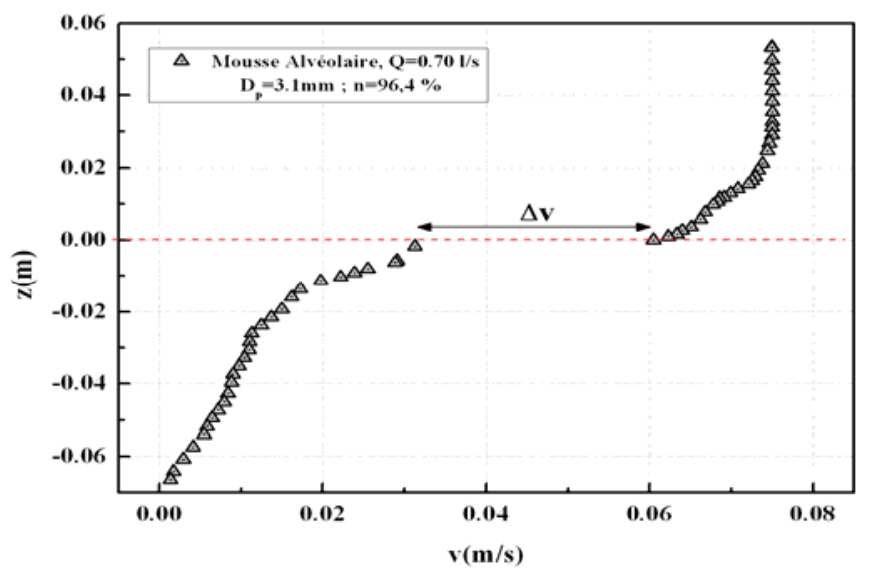

Figure 8. Détail de la discontinuité des vitesses à l'interface eau/sédiment.

\section{B.4. Étude plus spécifique dans le cas d'un fond sableux soumis à la houle dans la zone de swash}

Cette partie est détaillée dans la thèse d'État de K. Mihoubi dont j'ai assuré l'encadrement dans mon laboratoire à Caen. Le travail est complexe et fera l'objet d'une publication indépendante. Par contre une vidéo sera disponible lors de la présentation. Mais ici, il convient de limiter la publication à l'élément qui me semble important, celui de la diffusivité de la quantité de mouvement, car c'est cette grandeur qu'il convient de prendre en compte dans les études de l'érosion des plages sableuses sous l'action de la houle.

\section{B.5. Conclusion.}

Les résultats mis en évidence par la mesure des vitesses au sein de toute la masse fluide (écoulement libre et écoulement interstitiel) montrent que les modèles classiques de détermination de la contrainte de frottement $\tau_{\mathrm{p}}$ à la paroi ne sont pas bien adaptés car ils sont basés principalement sur les caractéristiques de l'écoulement libre sans prendre en compte la nature du sédiment, ses caractéristiques (porosité) et les caractéristiques de l'écoulement interstitiel (nombre de Reynolds des pores).

Cette contrainte de frottement $\tau_{\mathrm{p}}$ est associée au seuil de mouvement. Il nous semble donc important de la définir en prenant en compte, en plus des caractéristiques de l'écoulement libre, les caractéristiques du sédiment et surtout de l'écoulement interstitiel. Cet objectif était celui de nos prochains travaux. 


\section{XVI'̀mes Journées Nationales Génie Côtier - Génie Civil \\ Le Havre 2020}

En comparant ces résultats à ceux obtenus dans le cas du transfert de chaleur (BÉLORGEY \& TRINITE, 1979), nous pensons qu'il serait important de définir une grandeur associée au transfert de quantité de mouvement au sein de la veine fluide, analogue au transfert de l'enthalpie dans le cas de transfert thermique. Et d'associer les phénomènes turbulents au sein de la sous couche de régénérescence et les phénomènes de transfert lorsque deux corps sont mis en contact.

Lorsque les particules fluides arrivent à la paroi, elles transfèrent leur quantité de mouvement ou (et) leur chaleur. Et les équations se réduisent pour la chaleur :

$\frac{\partial \theta}{\partial t}=a \frac{\partial^{2} \theta}{\partial x^{2}}$

$a$ : étant la diffusivité thermique $a=\lambda / \rho C_{p}$.

Pour la quantité de mouvement :

$\frac{\partial \rho u}{\partial t}=v \frac{\partial^{2} \rho u}{\partial x^{2}}$

$v$, étant la viscosité. La solution de ces équations s'exprime sous la forme :

$X=X_{0}$ erf. $\left(\frac{x}{\sqrt{4 k t}}\right)$, avec $X=(\theta$ ou $\rho u)$ et $k=a$ ou $v$.

La densité de flux transféré est alors : $\varphi=X_{0} \frac{b}{\sqrt{\pi}} \cdot \frac{1}{\sqrt{t}}$.

Pour les temps de contact courts (turbulence) la densité de flux transféré est très grande.

Nous proposons donc, pour les problèmes d'érodabilité, de prendre en considération une grandeur sous la forme :

$b_{f l}=\sqrt{\mu \rho C_{i}^{2}}$

analogue à l'effusivité :

$b_{t h}=\sqrt{\lambda \rho C_{p}}$

\section{Références}

BÉLORGEY M., TRINITE M. (1979). Etude du flux à la paroi à l'aval d'une source linéaire de chaleur placée dans une couche limite turbulente se développant au-dessus d'une plaque plane lisse et isotherme. Letters in Heat and Mass Transfer, Vol. 6(1), pp 45-56. https://doi.org/10.1016/0094-4548(79)90030-4

BÉLORGEY M., LE BAS J. (1984). Mesure par Vélocimétrie Doppler Laser de la vitesse instantanée des particules fluides dans la houle produite en laboratoire. La Houille Blanche, n5, pp 363-368. https://doi.org/10.1051//hb/1984024 


\section{Thème 1 - Hydrodynamique côtière}

BÉLORGEY M., LE BAS J., GRANDJEAN A. (1989). Application of Laser Doppler Velocimetry to the study of turbulence generated by swell in the vicinity of walls or obstacles. Coastal Engineering, Vol. 13, pp 183-203. https://doi.org/10.1016/0378-3839(89)90023-9 BÉLORGEY M., BERGMAN H., DE GERLONI M., FRANCO L., PASSONI G. (2000). Perforated caisson breakwaters: Wave loads and hydraulic performance. Selection of Coastal Stuctures Congress Papers, Santander, Spain, 1999, A.A. Balkema Editor, Coastal Structures '99, Vol 2, pp 603-612.

FICHAUT B., HALLÉGOUËT B. (1989). Banneg, une île dans la tempête. Penn ar Bed, $\mathrm{n}^{\circ} 135$, pp 36-43.

FICHAUT B., SUANEZ S. (2005). Milieux littoraux nouvelles perspectives d'étude. Éditions 1'Harmattan, pp 53-74.

FICHAUT B., SUANEZ S. (2007). Les blocs cyclopéens de Banneg. Penn ar Bed, pp 13-24. hal-00192770

GUILCHER A. (1959). L'archipel de Molène (Finistère) Etude morphologique. Revue de géographie Physique et de Géologie.

MIHOUBI K., BÉLORGEY M., LEVACHER D, KETTAB A. (2008). Étude de la répartition des vitesses interstitielles au sein d'un lit perméable sous un écoulement à surface libre. Revue Paralia, Vol. 1, pp 1.1-1.14. https://doi.org/10.5150/revue-paralia.2008.001

\section{Annexe}

Image d'un bloc de roche projeté à $10 \mathrm{~m}$ en retrait du sommet de la falaise, le 31/10/05, (FICHAUT \& HALLÉGOUËT, (1989); FICHAUT \& SUANEZ (2007)).

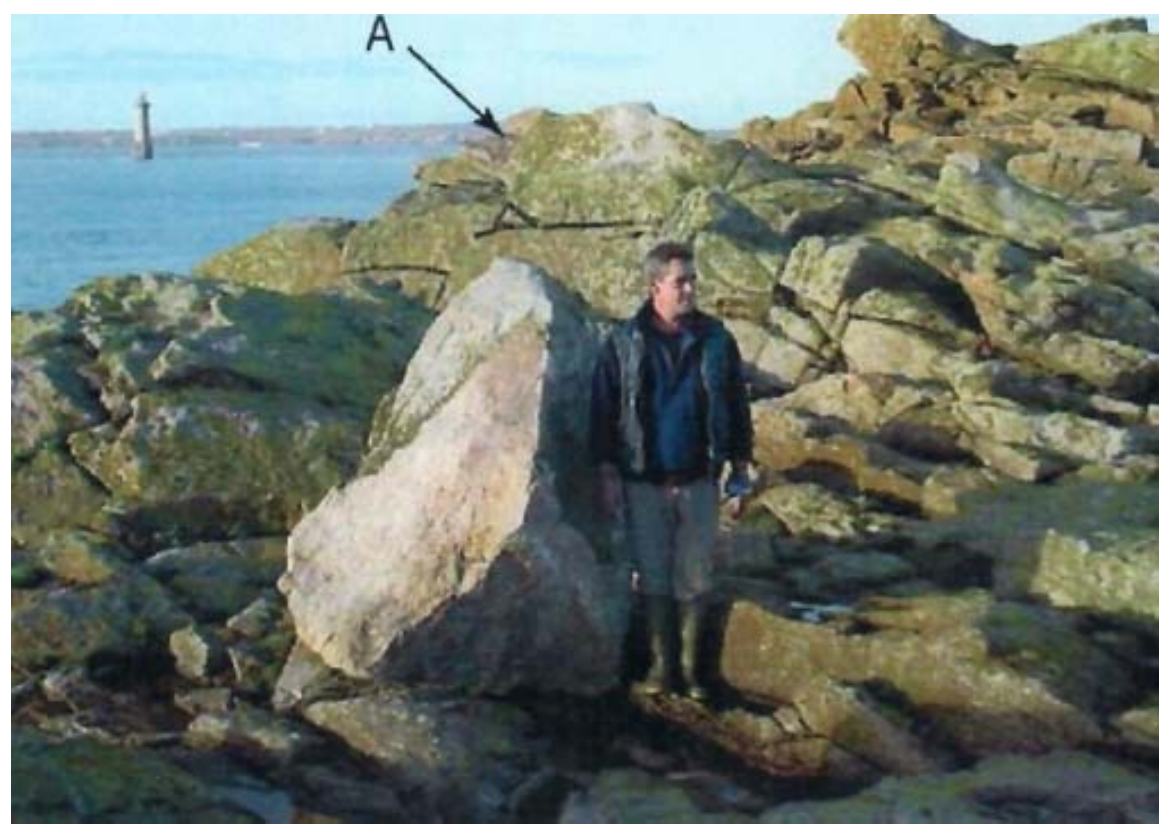

Figure A. Ile de Banneg, bloc projeté, FICHAUT \& SUANEZ (2005). 